\title{
Appropiate Initial Adenosine Dosage for Children with Supraventricular Tachycardia
}

\author{
Moises Rodriguez-Gonzalez ${ }^{{ }^{*}}$, MD, Ana Castellano-Martinez ${ }^{2}$, MD \\ ${ }^{1}$ Pediatric Cardiology Department of Hospital Universitario Puerta del Mar, Cadiz, Spain \\ ${ }^{2}$ Pediatric Department of Hospital Universitario Puerta del Mar, Cadiz, Spain
}

*Corresponding Author: Moises Rodriguez-Gonzalez, Pediatric Cardiology Department of Hospital Universitario Puerta del Mar, Cadiz, Spain, Email: doctormoisesrodriguez@gmail.com

\begin{abstract}
SVT can be defined as an abnormally rapid heart rhythm originating above the ventricles, often (but not always) with a narrow QRS complex on ECG. Re-entrant SVT is the most frequent mechanism of tachycardia in childhood (>90\%).Adenosine is widely recognized as the first line of pharmacologic treatment for SVT. Since, the initial clinical trials in late 1980s, the adequate dosage of adenosine have been questioned and current guidelines offer confusing advice about adenosine dosage in children, with very heterogeneous dosage recommendations. In this review of the pediatric literature we show that in several studies the optimal dose of adenosine is higher than the $0.1 \mathrm{mg} / \mathrm{kg}$ recommendation. Because adenosine is a drug with few adverse effects owing to its very short half-life, it seems advisable to recommend a higher initial dose, at least $200-300 \mathrm{mcg} / \mathrm{kg}$.
\end{abstract}

Keywords: Supraventricular tachycardia; Adenosine; Children; Antiarrhythmic drugs.

\section{SUPRAVENTRICULAR TACHYCARDIA (SVT)}

SVT can be defined as an abnormally rapid heart rhythm originating above the ventricles, often with a narrow QRS complex on ECG. SVT is the most common tachyarrhythmia requiring treatment in childhood, with an estimated incidence of $1 / 250$ to $1 / 1000$ children[1,2]. Re-entrant SVT is the most frequent mechanism of tachycardia in childhood (>90\%)[3]. In infants and children it is most commonly the result of electric conduction through accessory pathways between the atria and the ventricles (atrioventricular re-entrant tachycardia, mostly Wolff-Parkinson-White syndrome)[2]. In other cases, it is secondary to intranodal re-entry at the AV node, which is more common in adolescents and adults (atrioventricular nodal re-entrant tachycardia). Both mechanisms utilize AV node to allow the electrical impulse to go on repetitively between the ventricles and the atrium, resulting in tachycardia[1](Figure 1).

\section{Reentry Paroxysmal Supraventricular Tachycardia}

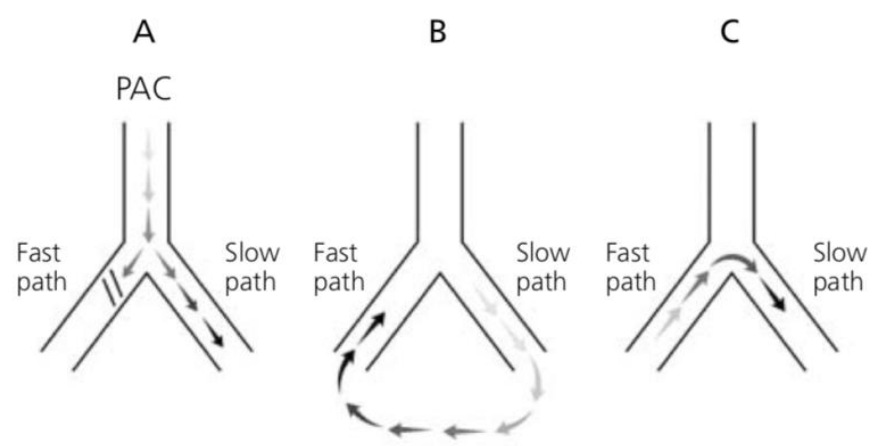

Figure1. Mechanism for reentrant paroxy smalsupraventriculartachycardia. (A) A premature atrial complex $(P A C)$ occurs and is blocked in a fast pathway, but it can propagate down the slower pathway. (B) By the time the electrical signal reaches the end of the slow pathway, the fast pathway has repolarized, and retrograde conduction of the wave occurs. $(C)$ The wave then returns down the slow pathway, setting up a closed circuit that is self-sustaining. 
The first step in the management of SVT is to determine whether the patient is hemo dynamically stable[4]. If SVT is sustained and there is any indication of instability electrical cardio version should be performed urgently. If not, conservative measures should be applied that include both non-pharmacologic (vagal manoeuvres) and pharmacologic measures[2]. Vagal manoeuvres are a number of physical manoeuvres increase the resistance of the $\mathrm{AV}$ node to transmit impulses (AV nodal block), principally through activation of the parasympathetic nervous system, conducted to the heart by the vague nerve.

\section{AdENOSINE}

Adenosine is widely recognized as the first line of pharmacologic treatment for SVT[4,5]. It is an endogenous purine nucleotide (6-amino-9beta-D-ribofuranosyl-9-H-purine) present in all cells of the body (Figure 2).[6,7] that has an important role in regulating the myocardial oxygen supply-demand balance. Adenosine achieves this through coronary vasodilation, decreasing myocardial contractility, and depressing automaticity and conduction within the sinus and atrioventricular (AV) nodes. [6,7].



Figure2. Adenosine molecular structure

As an antiarrhythmic agent, adenosine has several unique properties as it is an intermediate metabolite, it has a very short half-life (less than 1.5 seconds), its effects are mediated by specific membrane purine (cell surface A1 and A2 adenosine) receptors coupled to guanine nucleotide binding proteins ( $\mathrm{G}$ proteins), and it has site specific actions in the myocardium with important differential effects in supraventricular and ventricular tissue (Figure 3). Activation of cardiac A1 receptors has a myocardial depressant effect. A1 receptor activation mediates inhibition of cAMP-mediated calcium influx and enhances potassium conduction. As a result, there is (1) hyperpolarisation and resultant slowing of the sinus node, (2) depression of action potential in the $\mathrm{AV}$ node, (3) mild inhibition in inward calcium current (ICa) in atrial myocytes and (4) suppression of ICa current in ventricular myocytes, leading to negative dromotropic, chronotropic and inotropic effect. These mechanisms explain how adenosine terminates SVTs after intravenous infusion[6,7]. A2 receptors which are found in endothelial cells and vascular smooth muscle and are responsible for the enhancement of adenylylcylase activity and increased cyclic AMP which relaxes smooth muscle and produces vasodilation (Figure 3)[6,7].



Figure3. Adenosine cellular mechanisms of action 
The above cardiovascular effects make adenosine a very useful and effective agent in controlling SVT, and it is widely accepted as the first line treatment for rapid termination of the short-term episode of hemodynamically stable SVT in infants and children which have failed to respond to vagal manoeuvres.

\section{INITIAL DOSAGE OF ADENOSINE IN CHILDREN}

Adenosine is very effective in reverting SVT to sinus rhythm, terminating SVT in $90-99 \%$ of patients when administered [5,8,9]. However this response is usually achieved at various incremental doses, not at the initial dosage in children. Thus the initial dosage of adenosine in SVT is a highly controversial issue in pediatric patients. Early experimental work and guidelines suggested adenosine doses of 37.5$50 \mu \mathrm{g} / \mathrm{kg}[5,10,11]$. Later studies supported evidence of higher doses of adenosine for optimum results in children and showed that the mean effective dose was around 150 $\mathrm{mcg} / \mathrm{Kg}[8,12,13]$. Current guidelines offer confusing advice about adenosine dosage in children, with very heterogeneous dosage recommendations. The starting recommended dosage varies between $50-100 \quad \mathrm{mcg} / \mathrm{kg}$ increasing by $50-100 \mu \mathrm{g} / \mathrm{kg}$ every 2 min to a maximum dose of $300-500 \mu \mathrm{g} / \mathrm{kg}$ if no effect is seen. $[4,14]$.

The pediatric literature shows that these current recommended adenosine initial doses for acute management of SVT are ineffective in the vast majority of cases. Thus the reported response to initial recommended dosage of $50 \mathrm{mg} / \mathrm{Kg}$ and $100 \mathrm{mg} / \mathrm{Kg}$ is only $6-16 \%$ and $22-36 \%$ respectively $[9,13,15]$. This minimum effective dose approach is designed to minimize to minimize side effects, but it is important to bear in mind that if multiple doses are required to control SVT, it may result in increased exposure to the drug and possibly increased side effects. Consistent with this Losek et al. found that a higher total dose was needed when the first administered dose was low[15]. The most important implication of this too low starting dosage is that paediatricians could lose confidence in what is a very effective drug and also the delay of control for a potentially lifethreatening complication. For the daily clinical practice it is important to know that the maximum recommended dose for children of $500 \mathrm{mg} / \mathrm{kg}$ and neonates of $300 \mathrm{mg} / \mathrm{kg}$ is for a singly administered dose, not for the resulting cumulative dose after summing each dose of adenosine administered, so the cumulative dose should not precludes further administration.

A high initial adenosine dosage approach constitutes probably the best management for SVT in children. In all pediatric adenosine studies the average effective dose was consistently reported to be $>150 \mu \mathrm{g} / \mathrm{kg}$, so it seems that this could be the initial dosage $[8,9,12,13,16]$. The response to doses up to $200 \mu \mathrm{g} / \mathrm{kg}$ has been reported to be $53 \%-64 \%$, and the mean effective dose of adenosine to control SVT is reported to be 160 $\mathrm{mcg} / \mathrm{Kg}[8,12,13,16]$. Also a reduction in risk of unsuccessful cardioversion of $35 \%$ (95\% CI $28 \%$ to $43 \%$ ) using the higher adenosine dose has been demonstrated[9].

The initial adenosine dosage differs in small infants and children[1,13,16]. Also the average dose of adenosine required for tachycardia termination in children has been reported to be higher than in adults[13].A dose of $50 \mathrm{mg} / \mathrm{kg}$ is effective in $10 \%$ of infants and children and 100 $\mathrm{mg} / \mathrm{kg}$ is effective in $25 \%$ of infants and $50 \%$ of children. A dose of $150 \mathrm{mg} / \mathrm{kg}$ is more effective in children (around 80\%) than in infants (around $35 \%)[9,13,16]$. This difference may be explained by the difference in weight to body surface area ratio. Other possible explanations for the lower response in infants include smaller cannulae limiting the injection rate and the fact that babies tend to be more ill at presentation and may have prolonged circulation times[1]. Adenosine has a very short half-life of only 8$10 \mathrm{~s}$, so it is imperative that the drug is rapidly delivered to its intended site of action, the atrioventricular node and therefore adenosine has to be administered through a vein of good calibre, preferably in the upper limbs, as close as possible to the heart. The infusion rate will be the fastest possible, and the subsequent flushing with physiological saline must be performed to accelerate the arrival of the drug in the coronary circulation. The smaller gauged cannulae, which are frequently chosen when treating children in view of their smaller veins and in order to reduce the pain associated with cannula insertion, do not permit rapid delivery of adenosine and do not facilitate sufficient flow rates and therefore reduce the chance of cardioversion, necessitating repeated and higher dosing.

Finally high initial dosage of adenosine is a safe practice $[8,9,12,13,16]$. After intravenous (IV) administration, adenosine is almost completely eliminated after a single pass through the 
coronary circulation. It is rapidly taken up from plasma into red blood cells and blood vessel endothelial cells where it is converted to adenosine-5'-monophosphate, inosine, or both. In physiological concentrations, adenosine has a half-life of 0.6-1.5 seconds, but in therapeutic doses the effects persist for 20-30 seconds[2,13]. Thus although side effects are common (cumulative clinical side effects occur in about $30 \%$ of patients) they are usually transient and mild, and do not require clinical intervention. Side effects include flushing, headache, chest discomfort, cough, and occasionally hypotension. No significant or sustained adverse affects when giving adenosine in therapeutic doses are usually reported in children. Short autolimited runs of nonsustained ventricular tachycardia, sinus tachycardia, or atrial fibrillation may also be observed after termination of tachycardia, but only sporadic life-threatening tachyarrhythmia generated or accentuated by adenosine are reported[17]. In addition, transient sinus bradycardia and $\mathrm{AV}$ block may also occur. Prolonged sinus arrest or AV block is uncommon but is dose-dependent and is seen in patients with underlying conduction disturbances.

\section{Conclusions}

Although most patients with SVT have a mild course, a few may develop cardiac failure and cardiogenic shock if prolonged and/or not diagnosed. Adenosine is an effective medicine in treating SVT in children and the current recommended starting doses of adenosine seems to be low.

A review of the pediatric literature shows that in several studies the optimal dose of adenosine is higher than the $50-100 \quad \mathrm{mcg} / \mathrm{kg}$ recommendation. Because adenosine is a drug with few adverse effects owing to its very short half-life, it seems advisable to recommend a higher initial dose, at least $200-300 \mathrm{mcg} / \mathrm{kg}$.

The type of venous access; the speed of administration; the flushing of the line; age; and cardiovascular status, are important factors influencing in the efficacy of adenosine to abort SVT.

\section{REFERENCES}

[1] Connor S. Treatment of supraventriculartachycardia with adenosine in children: size does matter. Emergency Medicine Journal 2009;26:911-2. doi:10.1136/ emj.2008.069252.

[2] Ratnasamy C, Rossique-Gonzalez M, Young M-L. Pharmacologicaltherapy in childrenwith atrioventricular reentry: which drug?
CurrPharm Des 2008;14:753-61.

[3] Connor S. Treatment of supraventriculartachycardia with adenosine in children: size does matter. Emergency Medicine Journal 2009;26:911-2. doi:10.1136/emj.2008.069252.

[4] López-Herce J, Rodríguez Núñez A, Maconochie I, Van de Voorde P, Biarent D, Eich C, et al. [Current international recommendations for pediatric cardiopulmonary resuscitation: the European guidelines]. Emergencias 2017; 29:266-81.

[5] Sherwood MC, Lau KC, Sholler GF. Adenosine in the management of supraven tricular tachycardia in children. J Paediatr Child Health 1998; 34:53-6.

[6] Eltzschig HK. Adenosine: an old drug newly discovered. Anesthesiology 2009; 111:904-15. doi:10.1097/ALN.0b013e3181b060f2.

[7] Fredholm BB. Adenosine, an endogenous distresssignal, modulates tissue damage and repair. Cell Death Differ 2007; 14:1315-23. doi:10.1038/sj.cdd.4402132.

[8] Díaz-Parra S, Sánchez-Yañez P, ZabalaArgüelles I, Picazo-Angelin B, Conejo-Muñoz $\mathrm{L}$, Cuenca-Peiró V, et al. Use of adenosine in the treatment of supraventriculartachycardia in a pediatric emergency department. PediatrEmergCare 2014; 30:388-93. doi:10.1097/PEC.000 0000000000144.

[9] Quail MA, Till J. Question 3 Does a higherinitialdose of adenosineimprovecardioversionrates in supraventriculartachycardia? Archives of Disease in Childhood 2012; 97:177-9. doi:10.1136/ archdischild-2011-301079.

[10] Qureshi AU, Hyder SN, Sheikh AM, Sadiq M. Optimaldose of adenosine effective for supraventricular tachycardia in children. J CollPhysiciansSurgPak 2012; 22:648-51. doi:10.2012/JCPSP.648651.

[11] Till J, Shinebourne EA, Rigby ML, Clarke B, Ward DE, Rowland E. Efficacy and safety of adenosine in the treatment of supraventriculartachycardia in infants and children. Br Heart J 1989;62:204-11.

[12] Dadi G, Fink D, Weiser G. Highdoseadenosinefor refractory supra ventricular tachycardia: a case report and literature review. Cardiol Young 2017;27:981-4. doi:10.1017/ S1047951116002626.

[13] Dixon J. Guidelines and adenosine dosing in supraventriculartachycardia. Archives of Disease in Childhood 2005;90:1190-1. doi:10.1136/adc.2005.077636.

[14] de Caen AR, Berg MD, Chameides L, Gooden CK, Hickey RW, Scott HF, et al. Part 12: Pediatric Advanced Life Support: 2015 American Heart Association Guidelines Update 
for Cardiopulmonary Resuscitation and Emergency Cardiovascular Care (Reprint). Pediatrics 2015; 136 Suppl 2:S176-95. doi:10.1542/peds.2015-3373F.

[15] Losek JD, Endom E, Dietrich A, Stewart G, Zempsky W, Smith K. Adenosine and pediatric supraventriculartachycardia in the emergency department: multicenter study and review. Ann EmergMed 1999;33:185-91.

[16] Qureshi AU, Hyder SN, Sheikh AM, Sadiq M.
Optimaldose of adenosine effective for supraventri culartachycardia in children. $\mathbf{J}$ CollPhysiciansSurgPak 2012; 22:648-51. doi:10.2012 /JCPSP.648651.

[17] Singh V, Salehi N, Thakur RK. Adenosineinduced tachycardia acceleration: an unusual proarrhythmia. Case Reports 2015; 2015:bcr2014207823-3. doi:10.1136/bcr-2014207823

Citation: Moises Rodriguez-Gonzalez, Ana Castellano-Martinez. Appropiate Initial Adenosine Dosage for Children with Supraventricular Tachycardia. ARC Journal of Cardiology. 2017; 3(2) 16-20. doi: dx.doi.org/11.20431/2455-5991.0302003.

Copyright: (C) 2017 Authors. This is an open-access article distributed under the terms of the Creative Commons Attribution License, which permits unrestricted use, distribution, and reproduction in any medium, provided the original author and source are credited. 